\title{
Hybridized Model for Early Detection and Smart Monitoring of Forest Fire
}

\author{
Mohammed Anas El abbassi, Abdelilah Jilbab, Abdennaser Bourouhou \\ Electrical Engineering Research Laboratory \\ ENSET-Rabat, Mohammed V University in Rabat \\ Rabat, Morocco \\ elabbassi.3@gmail.com
}

\begin{abstract}
The demand for wireless sensor network technology has been increasingly needed in recent years for several major applications, including environmental monitoring, where nodes deployed in nature detect, process and transfer the environmental data in an autonomous way. However, the performance of early detection of the fire event after data fusion processed by this WSN's system will be less reliable since the majority of the other nodes do not detect the fire yet (at the beginning of event). That is why the present work is conducted. It proposes an intelligent strategy of data fusion of temperature and humidity sensors hybridized with an intelligent scanning technique. This model will allow the early detection of alarm from the beginning of fire event and guarantee a good monitoring of area state with an ongoing localization of fire zone. The result proves a very good performance in terms of reliability of the early detection and tracking fire propagation.
\end{abstract}

Keywords - Wireless sensor networks (WSN); early detection; data fusion; scanning technique; fire event; monitoring.

\section{Introduction}

As the exploitation of the wireless sensor networks [1] is strongly demanded in several fields, such as the environment (as discussed in [2]-[3]-[5]-[12]), the army and medicine...etc, some scientific researchers still lay their focus on the same direction in order to find promising resolutions to improve the performance of these sensors in terms of energy consumption, routing protocols, compression and transmission of distributed data, and collaborative signal processing which shapes our main interest, particularly, the processing of the multi-sensor data fusion.

Regarding the studies on the reliability of alerts, it is affirmed in several related works that the fusion of information multi-sensors plays a primordial role for a good reliability of alert. However, several fusion models (as in [4] - [5]) treat the alert issue based on the acquisition of data provided from the nodes deployed in the surveillance field, in the case of a fire event and at the very beginning, minorities of the nodes immediately detect the fire, while, the other nodes that are still far from the event, have not detected the fire yet. The system, then, starts the calculation based on all the collected data. Such reasoning requires sufficient propagation of the fire before it is detected by the system, which 
systematically generates an alert delay that can cause burns of large areas of the monitored environmental zone.

In addition, many of these proposed approaches of researchers (as in [4] and [5]), have not discussed the information filtering processing, which is an interesting operation that allows to avoid the impact of erroneous data on the processing of information fusion.

It is in this regard, the present work proposes an intelligent architecture of data fusion related with a scanning technique in order to reliably detect and locate the fire zone at the beginning of its propagation in the area. Such a model also takes into account the intelligent elimination of erroneous collected information.

\section{Proposed Work}

\subsection{Global Model Architecture}

For the discussion of the proposed model, let's consider that $\mathrm{n}$ nodes are dispersed in the monitored environment; these micro-sensors are capable to detect, collect and transfer the collected environmental data in an autonomous way (physical quantity of temperature and humidity) to the Sink point through a specific routing of the data. This Sink processes these data and transfers them to the control center where the central computer performs processing, fusing and decision-making operations.

This part intends to present a global fusion plan. This model reacts intelligently from the beginning of the fire with a minimum possible of propagation. Generally speaking, this model discusses a two data fusion modes hybridized with a scanning algorithm applied to the matrix of collected measurements. This proposed model contains global hierarchical steps (Fig. 1), each of which will be discussed separately in the present work.

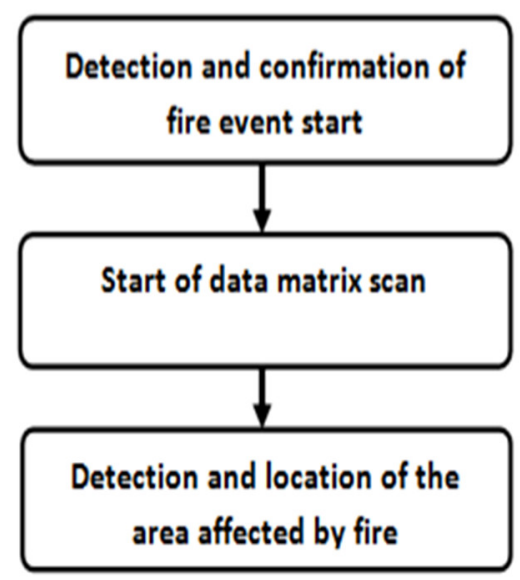

Figure 1 Global model process.

\subsection{Description of The Steps}

\subsubsection{Primary Fire Detection}

This part discusses a fusion approach that guarantees detection of fire from its first beginning by using at first the KNN classifier [6]-[7] for making a local decision in single node (first mode of data fusion), also, allowing to make a final reasoning of fire event based on collected samples of the neighboring nodes by 
Mohammed Anas El abbassi, Abdelilah Jilbab, Abdennaser Bourouhou, Hybridized Model for Early Detection and

Smart Monitoring of Forest Fire. Transactions on Machine Learning and Artificial Intelligence, Vol 5 No 4 August (2017); p: 364-372

using the Equal Gain Combiner $(E G C)$ fusion rule [8] chained to the $K N N$ classifier (second mode of data fusion).

\section{a) First Mode of Data Fusion}

This part focuses on the application of $K$-Nearest-Neighbors algorithm (KNN) [6]-[7] for evaluation of the combined data resulting from temperature and humidity measurements (see Fig.3) of single node. And to define the $K N N$ algorithm, the latter is among the most common methods for classification of the objects, applied since 1970's as a statistical estimation technique, this classification is based on a voting system of its $K$ nearest neighbors which are measured by the similarity function (or distance) relative to the target data [6]. This distance is usually measured by applying the Minkowski distance [9]-[10], described as following:

$$
M D=\left(\sum_{l=1}^{u}\left(\left|x_{l}-y_{l}\right|\right)^{p}\right)^{\frac{1}{p}}
$$

Where $x_{l}$ and $y_{l}$ are the coordinates of the two points $X$ and $Y$, while the parameters: $u$ and $p$ are selected by user ( $u=2, p=2$ in our approach).

Let $n=100$ nodes geographically dispersed in a surveillance zone with a surface of $90 \times 90 \mathrm{~m}^{2}$ where each known location node is distanced by $10 \mathrm{~m}$ from its nearest neighbors. These nodes are capable to measure the physical quantities of temperature and humidity.

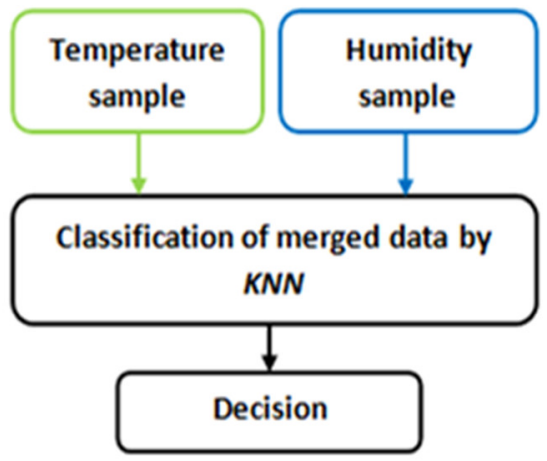

Figure 2 Local fusion and decision process inside node (First mode of data fusion).

Therefore, to apply this local data fusion operation by using $K N N$ (as shown in Fig.2), we have in the same node $C_{x}$ ( $\mathrm{x}$ is an index, $\left.\mathrm{x} \in[1 ; 100]\right)$, a case of collection of two physical quantities samples of temperature type denoted $T s_{x}$, and of humidity type denoted $H s_{x}$. Given that $D_{\text {Local }}\left(H s_{x}, T s_{x}\right)$ represents a result point based on these two coordinate samples where each combined $D_{\text {Local }}\left(H s_{x}, T s_{x}\right)$ must be processed and assigned to one of two clusters : \{Fire Cluster\} and \{None Fire Cluster\} as described in Fig.3. 


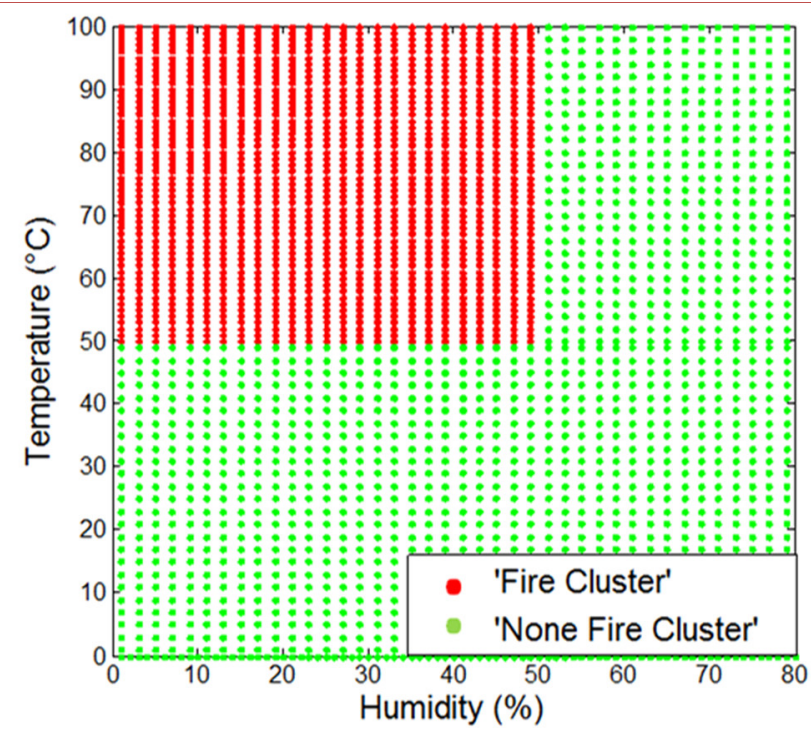

Figure 3 Definition of clusters regions according to temperature and humidity ranges.

As you can see, the previous clusters which are defined on two axes (Humidity and Temperature), express opposite hypotheses, their intervals in both axes, are carefully chosen by the user, taking into account the favorable conditions that generate the fire and also the climate of this geographical area being monitored. Note that these clusters are stored on the database and used as basic information on which $K N N$ classifier can refer to decide.

According to Fig.3, the D Local $x$ point expresses the danger state if it's assigned to \{Fire Cluster\}. Until now, as mentioned, it is information considered independent and specific to a single node. And to avoid any errors or malfunction or false testimony from a failing node, as a node detects such an alarm, the system reacts and begins to seek confirmation of the information by the occurrence of the nearest neighboring node (Fig. 4). At this stage, an analysis of fusion operation of these samples including the sample of the first sensor is initiated in order to confirm the presence or none of the fire, this new operation of data fusion called a second mode of data fusion that will be discussed in the next section.

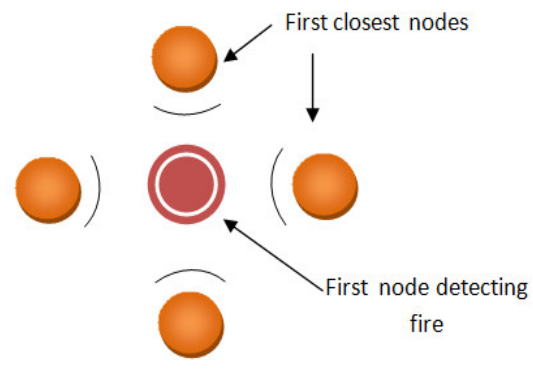

Figure 4 Example of the first node with its four neighboring nodes detecting the fire $(N=5)$ 


\section{b) Second Mode of Data Fusion}

Let $M D(2)$, a matrix of the local estimation samples denoted $D p_{i}(i=1,2 \ldots n)$ provided by $n=100$ nodes, where $D p=1$ represents a 'fire state' while $D p=0$ represents a 'none fire state', those decisions are collected for each $t=\tau$.

$$
M D=\left[\begin{array}{ccc}
D p_{1} & \cdots & D p_{10} \\
\vdots & \ddots & \vdots \\
D p_{91} & \cdots & D p_{100}
\end{array}\right]
$$

We take the case of detection of the first node $C_{x}$ located inside the matrix, which means that $D p_{x}=1$ as already mentioned. In this respect, the data fusion based on this sensor and the four surrounding neighbors is established to testify about the existence of fire or none. The proposed processing of the second mode of data fusion has been broken into phases which have been illustrated in the following Fig.5

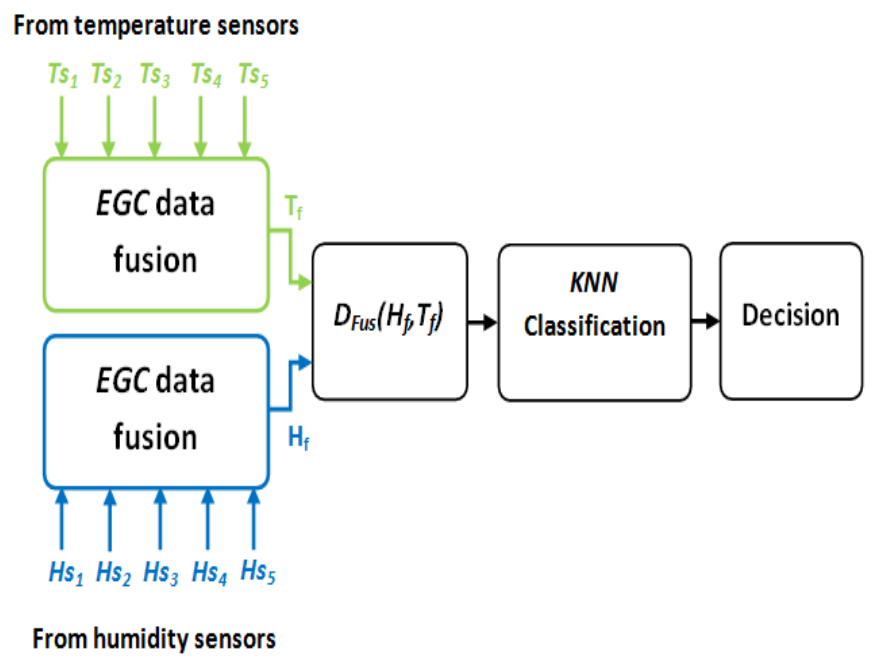

Figure 5 The proposed process of the second mode of data fusion over five nodes.

The first stage processes the combination of measures shipped from sensors of the same category by applying the $E G C$ fusion rule [8], the latter is among the most common algorithms of data fusion, it has thus shown its effectiveness in terms of speed in processing and its reliability of output with a minimum amount of information required, more than that, compared to some algorithms as mentioned in [8], it is considered robust and stable for a wide range of $S N R$.

At the output of the EGC blocks, we find ourselves with two fused samples of temperature and humidity denoted $T_{f x}$ and $H_{f x}$, the latter are subsequently the coordinates of a combined sample denoted $D_{F u s x}\left(H_{f x}\right.$, $\left.T_{f_{x}}\right)$, which is acted as a target data to the $K N N$ algorithm, this one, classifies the new data given the same reasoning previously discussed in the local fusion with a slight change in the boundaries of the intervals of the two clusters, this change in limits is taken into account in this mode of fusion in order to guarantee the correct decision based on a majority of the nodes among $N\left(N_{\max }=5\right)$ which provides measurements belonging in the same hypothesis. The output of the processing translates the final decision noted $F_{D x}$, which estimates the state of the area covered by these nodes. 
Parallel processing begins on the matrix zone (Fig.6) after detecting the fire by the first node $C_{x}$, as already mentioned, the system takes the state of solidification of the information by application of data fusion with the neighboring nodes, this algorithm takes into account the location of the first node detecting the alarm with their neighbors situated in this matrix zone, consequently, the number $N$ of the nodes varies automatically between 3 and 5 as described in Fig. 6 .

According to Fig. 6 , the primary analysis fusion can be established on the basis of a number $N$ of nodes that are located in one of the 3 mentioned regions. The first region, which is presented inside the matrix, processes the data fusion of the first node with its four neighbors. The second region comprises the four matrix edges (without corners) where the data fusion of four nodes is initiated. The third region includes the four corners of the area with an established data fusion of three nodes.

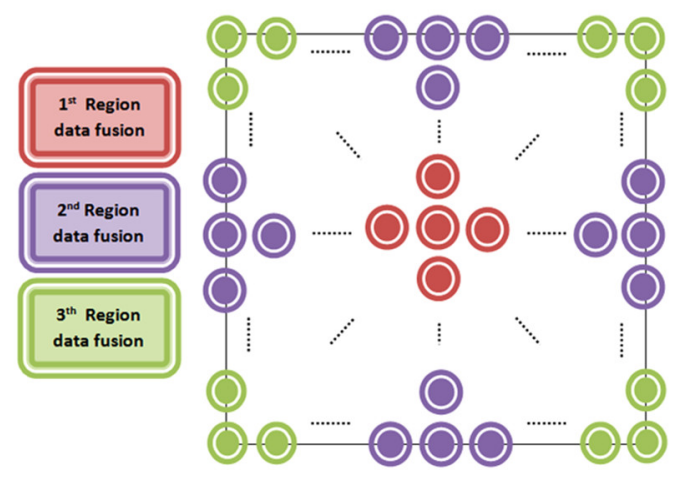

Figure 6 Definition of three different regions of data fusion in the matrix zone.

\subsubsection{Scanning of The Matrix Data}

After confirming a beginning of a fire start by the proposed technique above, it becomes essential to disclose continually the areas affected during the propagation of the fire. The scanning operation of the matrix zone is performed from the first affected area by a reasoning of data fusion of $N$ nodes, the same operation is conducted again after incrementing or decrementing the scanning process in the matrix.

The scan, in this case, divides the matrix from the first detection point into two zones namely: A and B as shown in Fig. 7 (it may be just a single zone of the entire matrix if the fire starts from one of the four regions corners). Thus, this scan starts from the same detecting point and scans these two zones to the end points taking into account that each new data fusion is processed based on a number $N(3,4$, or 5 nodes) depending on the location of the fusion operation on the matrix during scanning.

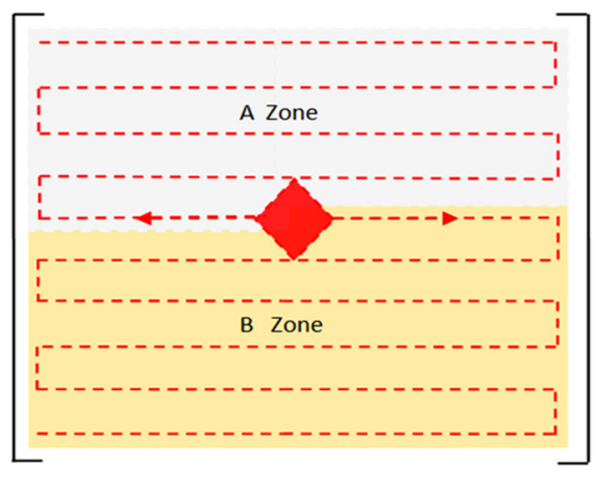

Figure 7 Scanning of the data matrix in two zones A and B (case of first detection inside the matrix zone). 
On the one hand, this operation contributes to identifying the sensors that detect the fire and to avoiding sensors providing incorrect data or sensors that are located in an area that is still intact. On the other hand, it allows determining the direction and the extent of the propagation of the fire and also monitoring the surface of the burned zone. This reasoning will be described in the next section.

\subsubsection{Detection, Location and Monitoring of The Affected Area.}

At the end of the scan, a new matrix of final binary decisions denoted $M_{\text {ext }}(3)$ is extracted. Through this matrix, we can actually illustrate the state of each part of the zone. Obviously, this illustration comes after having decided (fire or none-fire) based on each point of $M_{\text {ext }}$, where each point translates the state of the local zone under the surveillance field of $N$ neighboring nodes after data fusion. Knowing that the system already notes the location of each sensor node, it is easy to identify in this case the zones affected by the fire.

$$
M_{e x t}=\left[\begin{array}{ccc}
F_{D 1} & \cdots & F_{D 10} \\
\vdots & \ddots & \vdots \\
F_{D 91} & \cdots & F_{D 100}
\end{array}\right]
$$

In order to trace the direction of propagation of the fire, the centroid that is based on the nodes responsible for the positive decisions (fire event), is calculated at each new iteration of the scan. This centroid is updated at each scanning operation, as shown in Fig.7, noting that each calculated centroid becomes the starting point for the new scan processing.

\subsection{Simulation Results}

Here is an example of a simulation of fire propagation in a square matrix surface monitored by 100 nodes, after detecting the first fire event. The system begins scanning iterations and makes a continuous update of the affected area with tracing fire propagation path, as illustrated in Fig.8.

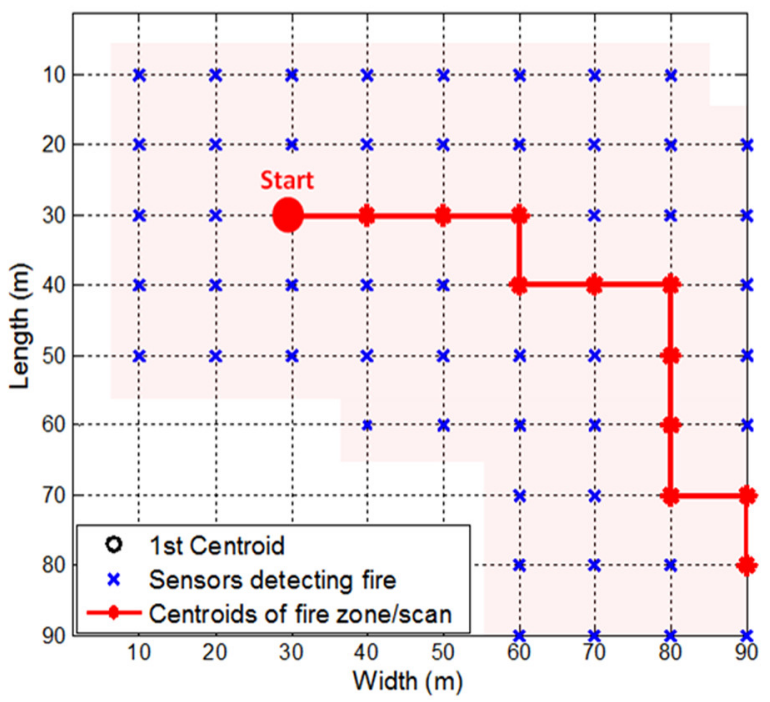

Figure 8 Monitoring of the affected area with propagation path tracing. (Example of area burning under 62 nodes detecting fire, while the area monitored by other 38 nodes is still intact). 
And, to evaluate the alarm response time, let's consider another case where the fire propagates on a surface of a circle the center of which is located by the first detector node and has a radius that increases by $1 \mathrm{~m} / \mathrm{s}$. The response of alarm detection will be illustrated in Fig.9.

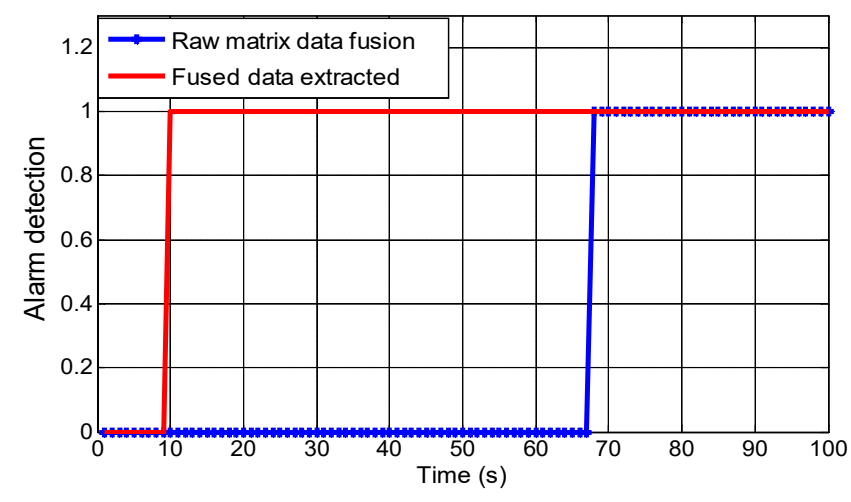

Figure 9 Responses comparison of alarm detection between the proposed model of data fusion and the raw data fusion of the entire matrix samples.

Fig.9 shows the positive response of the proposed method (in red line), where the alarm is triggered after a period of $10 \mathrm{~s}$, which coincides with a radius of $10 \mathrm{~m}$. The latter is reasoned by the distance fixed between the first detector and its neighbors. On the other hand, the alarm of a data fusion response of the matrix of the raw samples (in blue line) is triggered after a delay time of $67 \mathrm{~s}$ which coincides with $67 \mathrm{~m}$ of the radius of the burned zone circle; therefore, it can really causes great environmental damage. On the premise of this reasoning, the proposed method shows its efficiency in terms of vigilance and rapidity of alert response.

\section{Conclusion}

In this work, we have presented a new approach of multi-sensor data fusion with two types of physical quantities (temperature and humidity) integrated in nodes deployed in a surveillance environmental area. It has also presented an intelligent strategy of hybridization between a data fusion operation and a scanning technique. In brief, this approach aims at making the early detection of fire event more reliable, with a minimum possible of fire damage while maintaining monitoring of the area state and defining a continuous tracing the fire propagation path.

The simulation results of the proposed approach show a very effective performance of early detection and prove efficient monitoring and accurate localization of the affected area.

We have considered using fast response algorithms (such as the $K N N$ classifier and the $E G C$ fusion rule), which are based on a simple arithmetic calculation that allowed for a flexible processing with a low energy consumption in WSN's system.

\section{REFERENCES}

[1] K. Sohraby, D. Minoli, T. Znati ." Wireless sensor networks: technology, protocols, and applications", John Wiley \& Sons (2007). 
Mohammed Anas El abbassi, Abdelilah Jilbab, Abdennaser Bourouhou, Hybridized Model for Early Detection and Smart Monitoring of Forest Fire. Transactions on Machine Learning and Artificial Intelligence, Vol 5 No 4 August (2017); p: 364-372

[2] M. Hefeeda,M. Bagheri,"Forest Fire Modeling and Early Detection using Wireless Sensor Networks", Ad Hoc \& Sensor Wireless Networks Vol. 7, pp. 169-224,2009.

[3] E. Sisinni, A. Depari, A. Flammini," Design and implementation of a wireless sensor network for temperature sensing in hostile environments", Sensors and Actuators A: Physical, vol.237, pp. 47-55, 1 January 2016.

[4] E. Zervas, A. Mpimpoudis, C. Anagnostopoulos, O. Sekkas, and S. Hadjiefthymiades,"Multisensor data fusion for fire detection", Information Fusion ,vol.12 :150-159, 2011.

[5] M.A.El abbassi, A.Jilbab,A.Bourouhou,"A robust model of multi-sensor data fusion applied in wireless sensor networks for fire detection",International Review on Modelling and Simulation (IREMOS), vol.9, N³,pp.173-180, Vol. 9, N. 3,pp 173-180, June 2016.

[6] Massart, D.L. "Alternative k-nearest neighbour rules in supervised pattern recognition: Part 1. k-nearest neighbour classification by using alternative voting rules." Anal. Chim.Acta 136, 15-27, January 1982.

[7] Vijay Kotu, Bala Deshpande, “Predictive Analytics and Data Mining,” ,chapitre 4, Pages 63-163 , 2015.

[8] R. Niu ,B. Chen, P.K. Varshney," Fusion of decisions transmitted over Rayleigh fading channels in wireless sensor networks “,IEEE Transactions on signal processing,Vol.54,NO.3,March 2006.

Teknomo, $\quad$ Kardi(2015),

http://people.revoledu.com/kardi/tutorial/Similarity/MinkowskiDistance.html ,(2015).

[10] M.A.El abbassi, A.Jilbab,A.Bourouhou," Detection model based on multi-sensor data for early fire prevention ", IEEE ICEIT tangier Morocco, pp.214 - 218. 2016.

[11] B. Khaleghi, A. Khamis, F.O. Karray, S.N. Razavi, "Multisensor data fusion :A review of state-of-the-art, Information fusion", vol 14: 28-44,2013.

[12] M.A.El abbassi, A.Jilbab, A.Bourouhou," Fusion des données dans les réseaux de capteurs sans fils pour la protection de l’environnement “, TELECOM'2015 \& 9ème JFMMA, Meknès- Maroc, 2015. 